\title{
Fabrication of highly ordered arrays of nanoparticles by
}

\section{mechanical process}

\author{
H. Ito ${ }^{1}$ and S. Nomura ${ }^{1,2}$ \\ ${ }^{1}$ Institute of Physics, University of Tsukuba, 1-1-1, Tennodai, Tsukuba, Ibaraki 305-8577, Japan. \\ ${ }^{2}$ CREST, Japan Science and Technology Corporation, Honcho, Kawaguchi 332-0012, Japan.
}

We have developed a new fabrication method of nanoparticles utilizing a mechanical rubbing process. Highly ordered arrays of Au nanoparticles with diameter of $60 \mathrm{~nm}$ have been fabricated on the porous anodic alumina template. Pressure measurements during the mechanical rubbing process confirmed that these nanoparticles have been formed only by mechanical separating off process. Our method can be applied to fabricate nanocapsules and has a wide range of applications for materials which have never been able to be controlled in nanoscale.

KEYWORDS: nanoparticles, nanocapsules, nanocapsules, self-assemble, porous alumina

H. Ito and S. Nomura, Fabrication of highly ordered arrays of nanoparticles by mechanical process. 
Fabrication methods of nanoparticles are conventionally categorized into the build-up process and the break-down process. Nanoparticles synthesized by the build-up process such as vapor-phase deposition process $^{1-3)}$ and wet chemistry process ${ }^{4-6)}$ utilize nucleation followed by growth to a required size. In contrast, the break-down process is a method where nanoparticles are fabricated by mechanically crushing bulk materials. The break-down process has advantages over the build-up process in that this process does not utilize the phase transition of the raw material accompanied by the temperature change or the chemical reaction in forming nanoparticles, which enables us to choose a wide variety of materials for nanoparticles. However, there are a few draw-backs of the conventional break-down process such as the difficulty in controlling the shape of nanoparticles, and the lower bound of the average size of nanoparticles of about a few hundred nanometers. Wet jet mill ${ }^{7)}$ and high-energy ball milling $^{8,9)}$ have been developed to fabricate smaller nanoparticles, but the shape of nanoparticles are not as uniform as those fabricated by the build-up process. Because the application areas of nanoparticles have recently been drastically expanding both in the fields of application and scientific research, a fabrication method of nanoparticles is required to meet the demanding requirements of both the high controllability of the shape and the broad range of selection of raw materials for nanoparticles.

In this paper, we report a simple technique for the fabrication of nanoparticles which has advantages of both the build-up process and the break-down process. We have developed a new fabrication method of nanoparticles utilizing a "rubbing" process. First a template with nanoholes is prepared either by the anodic oxidation technique ${ }^{10-12)}$, or by the electron beam lithography, and so on. The materials of nanoparticles are put on the template either by the vacuum deposition, or by the spin coating, and so on. In the rubbing process as shown schematically in Fig. 1, nanoparticles are formed by pressing the material into nanoholes by sliding a pud on the template. This method utilizes only a physically separating off process. Rubbing process may be classified into a break-down process by the conventional classification. But the accuracy in controlling the shape of nanoparticles is dramatically improved by using a template with a highly ordered nanohole array as compared with the conventional break-down process. Our experiment has demonstrated successful fabrication of highly ordered arrays 
of Au nanoparticles with a diameter of $60 \mathrm{~nm}$. Mechanical process was previously utilized as control of the feature of nanoscale structure as a fabrication method of a fiber probe for near-field scanning optical microscopy ${ }^{13)}$. A tapered fiber probe was coated with a Au film using a sputtering method. An aperture of the Au film with a diameter of $30 \mathrm{~nm}$ was created by pounding the probe apex against the flat surface.

We used porous anodic alumina (PA) as a template. PA has ordered honeycomb structure ${ }^{14,15)}$, where nanoholes are arranged in a triangular lattice by self-assembling. Arrays of nanoholes with spacing ranging from 10 to $500 \mathrm{~nm}$ can be grown by simply varying the anode voltage from 4 to $200 \mathrm{~V}^{10-12)}$. We prepared a highly ordered PA template by using two-step anodic oxidation by the condition as described in the refs. 10 and 11 . First a $0.5 \mathrm{~mm}$ thick aluminum plate (99.999\%) was anodized in $0.3 \mathrm{M}$ oxalic acid at $40 \mathrm{~V}$ at $0^{\circ} \mathrm{C}$ for 6 hours. A PA template with disorder areas formed by the first anodization was removed in an etchant composed of phosphoric acid (6 wt\%) and chromic acid $(1.8 \mathrm{wt} \%)$ at $60^{\circ} \mathrm{C}$ for 60 min. A highly ordered PA template was formed by the second anodization for 20 min under the same condition as the first anodization. The nanoholes were expanded to $60 \mathrm{~nm}$ at $30^{\circ} \mathrm{C}$ for $20 \mathrm{~min}$ in the same etchant as we used for the removal of the PA layer by the first anodization. The fabricated nanostructures were observed by a scanning electron microscope (SEM). Figure. 2(a) shows a SEM image of the surface of a PA template. The PA template was boiled up in acetone, isopropanol and deionized water. We chose Au as material of nanoparticles. We deposited a Au film on a PA template with the thickness of $50 \mathrm{~nm}$, of which geometric form is a triangular lattice without covering nanoholes of a PA template as shown in Fig. 2(b). We chose a stainless-steel ball with a diameter of $0.5 \mathrm{~mm}$ as a pad to be used in the rubbing process. In this process, the stainless-steel ball was rolled on the PA template and mechanical force was applied perpendicular to the surface of the PA template. During this process, magnitude of the force applied to the PA template was monitored.

Figure 2(c) shows a SEM image of the synthesized Au nanoparticles, which were obtained by rubbing a Au film with 50 nm-thick deposited on a template at $150 \mathrm{MPa}$. It can be seen that the Au nanoparticles are separated each other. Ordered arrays of Au nanoparticles with uniform size were obtained in the area larger than $1 \mathrm{~mm} \times 1 \mathrm{~mm}$.

H. Ito and S. Nomura, Fabrication of highly ordered arrays of nanoparticles by mechanical process. 
To investigate the deformation of the pressed material of nanoparticles, we varied the pressures perpendicular to the template during the rubbing process. A PA template with a $50 \mathrm{~nm}$ Au film was used as in the case of Fig. 2(b). Figure 3(a) shows the pressure as a function of the applied force perpendicular to the PA template. The pressure was calculated by measuring the contacted area and the force by assuming that the pressure is homogeneous in the contacted area. The measured pressure is proportional to the applied force up to $0.78 \mathrm{~N}$. At $1.77 \mathrm{~N}$, however, the pressure decreases because of the break down of the PA template. We observed this sample by a SEM, and found many cracks on the surface of the template, and the expanded contact area. In the following experiments, the perpendicular force was kept small enough not to damage the templates. Figure 3(b) shows a SEM image of Au nanostructures rubbed depending on the applied pressure. The diameter of the holes of the Au lattice is smaller for higher pressure. At $112 \mathrm{MPa}$, the surface of the PA template is seen between the Au nanoparticles showing that the $\mathrm{Au}$ nanoparticles are separated by pressing the $\mathrm{Au}$ film into the nanoholes by the rubbing process. From this experiment, it is confirmed that the formation of the nanoparticles as shown in Fig. 2(c) cannot be explained either by diffusion, wetting, or shadowing during deposition. A simple mechanism of the rubbing process as presented in Fig. 1 explains the formation process of the nanoparticles.

Figure 4 shows heat-treated $\mathrm{Au}$ nanoparticles, which are obtained by thermal annealing of the samples fabricated by the same process as in Fig. 2(c). Annealing was performed in N2 atmosphere at $600{ }^{\circ} \mathrm{C}$ for 5 min. Comparison of the Au nanoparticles before annealing [Fig. 2(c)] and after annealing [Fig. 4] shows that the annealed Au nanoparticles have more rounded shape and distinct outline. If the neighboring Au nanoparticles slightly touch each other before annealing, it is anticipated that the $\mathrm{Au}$ nanoparticles will fuse during the annealing process. In fact this fusion occurred by annealing the Au nanoparticles rubbed at $105 \mathrm{MPa}$. However, at $150 \mathrm{MPa}$, the Au nanoparticles after annealing were separated without fusion, and the arrangement of the nanoparticles were unchanged as shown in Fig. 4.

Our method can also be applied to fabricate nanocapsules. Here, we propose a method to fabricate nanocapsules. Test tube shaped nanostructures "nano test tubes” are prepared by forming a film with 
uniform thickness on the inner side of nanoholes of the PA template. For example, polymer nanotubes arrays are fabricated by a wetting process ${ }^{16)}$, and $\mathrm{ZnO}$ nanotubes arrays are fabricated by a sol-gel template process ${ }^{17)}$. A gold plug can be put on each nano test tubes by applying the rubbing process. Nanocapsules can be fabricated by the following procedures; First, fabricate the nano test tubes, second, filling the material into the nano test tubes, and third, putting plugs on the nano test tubes by using the rubbing process. It differs from the conventional process in that the shell material is coated around the core material. Therefore, even very soft materials such as liquid can also be capsuled. This process does not involve the phase transition of the raw material accompanied by the temperature change or the chemical reaction in forming nanocapsules. Therefore, it is possible to capsule proteins or cells which are sensitive to the changes in the environment. Separation of nanocapsules from the nanoholes of the PA template may be performed by selective etching of PA by using mixture of phosphoric acid (6 wt \%) and chromic acid (1.8 wt \%).

In summary, we propose a "rubbing process" as a general fabrication method of nanoparticles. We demonstrate successful fabrication of highly ordered arrays of Au nanoparticles with a uniform diameter by utilizing only a mechanical separating off process. We also show that our method can be applied to plug individual nanoholes of the PA template to form "nanocapsules". Our method has applications for wide range of materials which have never been able to be controlled in nanoscale. ${ }^{18)}$

ACKNOWLEDGMENT: We would like to thank Prof. Y. Ootuka for fruitful discussion and encouragement. This study was partly supported by the Gant-in-Aid for Scientific Research from Japan Society for the Promotion of Science, and by the University of Tsukuba Nano-science Special Project.

H. Ito and S. Nomura, Fabrication of highly ordered arrays of nanoparticles by mechanical process. 


\section{REFERENCES:}

1) P.C. Sercel, W.A. Saunders, H.A. Atwater, K.J. Vahala and R.C. Flagan: Appl. Phys. Lett. 61 (1992) 696.

2) N. Suzuki, T. Makino, Y. Yamada, and T. Yoshida: Appl. Phys. Lett. 78 (2001) 2043.

3) R. G. Osifchin, W. J. Mahoney, J. D. Bielefeld, R. P. Andres, J. I. Henderson and C. P. Kubiak: Superlattices Microstruct. 18 (1995) 283.

4) R. N. Bhargava and D. Gallagher: Phys. Rev. Lett. 72 (1994) 416.

5) K. Kurihara: J. Am. Chem. Soc. 105 (1983) 2574.

6) A. A. Guzelian, U. Banin, A. V. Kadavanich, X. Peng, and A. P. Alivisatos: Appl. Phys. Lett. 69 (1996) 1432.

7) H. Kezuka, Z. Xi, Y. Miyanaga and C. Kan-nari: Thin Solid Films 281 (1996) Issues 1-2, 584.

8) Y. Liu, J. Zhang, L. Yu, G. Jia, C. Jing, and S. Cao: J. Magn. Magn. Mater. 285 (2005) 138.

9) T. P. Yadav, N. K. Mukhopadhyay, R. S. Tiwari and O. N. Srivastava: Materials Science and Engineering A 393 (2005) 366.

10) H. Masuda and M. Satoh: Jpn. J. Appl. Phys. 35 (1996) L126.

11) A. P. Li, F. Mu“ller, A. Birner, K. Nielsch, and U. Go“sele: J. Appl. Phys. 84 (1998) 6023.

12) H. D. L. Lira and R. Paterson: J. Membr. Sci. 206 (2002) 375.

13) K. Matsuda, T. Saiki, S. Nomura, M. Mihara and Y. Aoyagi: Appl. Phys. Lett. 81 (2002) 2291.

14) F. Keller, M. S. Hunter, and D. L. Robinson: J. Electrochem. Soc. 100 (1953) 411.

15) J. P. O’Sullivan, and G. C. Wood: Proc. R. Soc. London A 317 (1970) 511. 
16) M. Steinhart, J. H. Wendorff, A. Greiner, R. B. Wehrspohn,K. Nielsch, J. Schilling, J. Choi, and U. Go“sele: Science 296 (2002) 1997.

17) G. S. Wu, T. Xie, X. Y. Yuan, Y. Li, L. Yang, Y. H. Xiao, and L. D. Zhang: Solid State Commun. 134 (2005) 485.

18) H. Ito and S. Nomura: PCT/JP2005/009990.

H. Ito and S. Nomura, Fabrication of highly ordered arrays of nanoparticles by mechanical process. 


\section{FIGURE CAPTIONS:}

Figure 1. Schematic illustration of a "rubbing" process. A metal film is deposited on a PA template with nanoholes. Nanoparticles are formed by pressing the deposited film into the nanoholes by sliding a rubbing pud on the surface of the PA template.

Figure 2. SEM image of the surface of a PA template at series of processes. (a) (Preparing a template) the bright lattice is a PA template, and the dark areas are nanoholes. (b) (Before rubbing process) A Au film is deposited on a PA surface with the thickness of $50 \mathrm{~nm}$. A Au film is brighter than a PA surface. (c) (After rubbing process) White disc-shape particles are Au nanoparticles which are fabricated by rubbing at $150 \mathrm{MPa}$.

Figure 3. (a) Pressure dependence of the force perpendicular to the substrate in the rubbing process. The pressure is proportional to the applied force up to $0.78 \mathrm{~N}$. At $1.77 \mathrm{~N}$ the pressure decreases because of the break down of the PA template. (b) The SEM image of Au nanostructures on a PA template before rubbing and after rubbing at pressures between $74 \mathrm{MPa}$ and $112 \mathrm{MPa}$.

Figure 4. The SEM image of annealed Au nanoparticles in $\mathrm{N}_{2}$ atmosphere at $600{ }^{\circ} \mathrm{C}$ for $5 \mathrm{~min}$. Au nanoparticles before annealing are formed at the same condition as in Fig. 2(c).

H. Ito and S. Nomura, Fabrication of highly ordered arrays of nanoparticles by mechanical process. 
Fig. 1 (double sized)

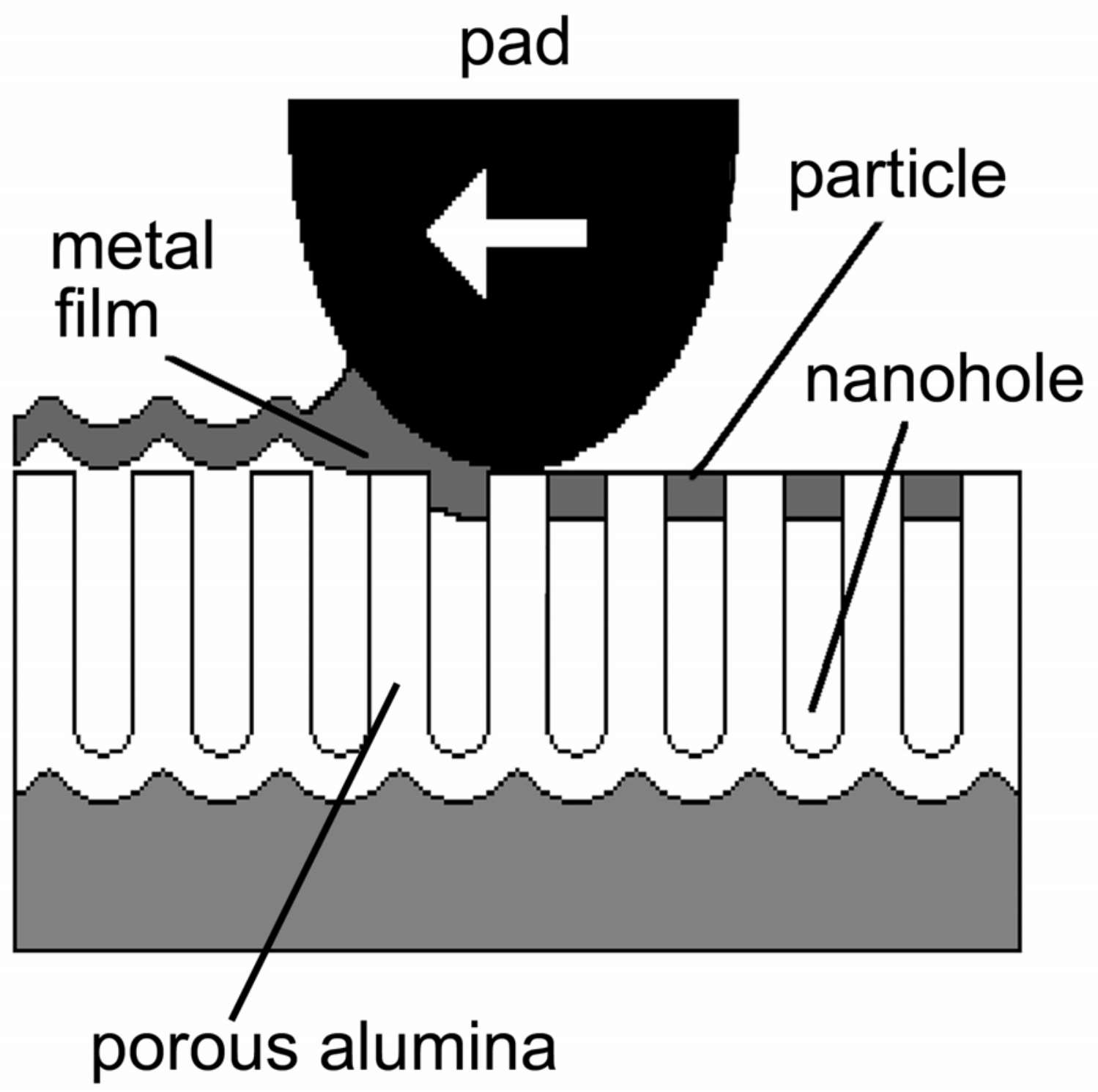

H. Ito and S. Nomura, Fabrication of highly ordered arrays of nanoparticles by mechanical process. 
Fig. 2
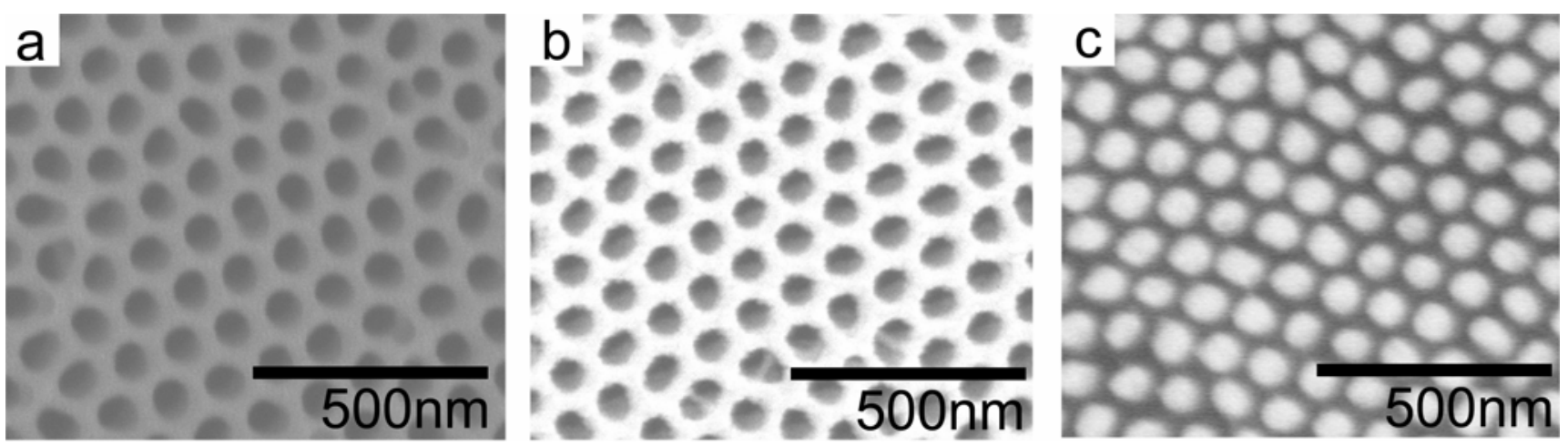

H. Ito and S. Nomura, Fabrication of highly ordered arrays of nanoparticles by mechanical process. 
Fig. 3 (double sized)

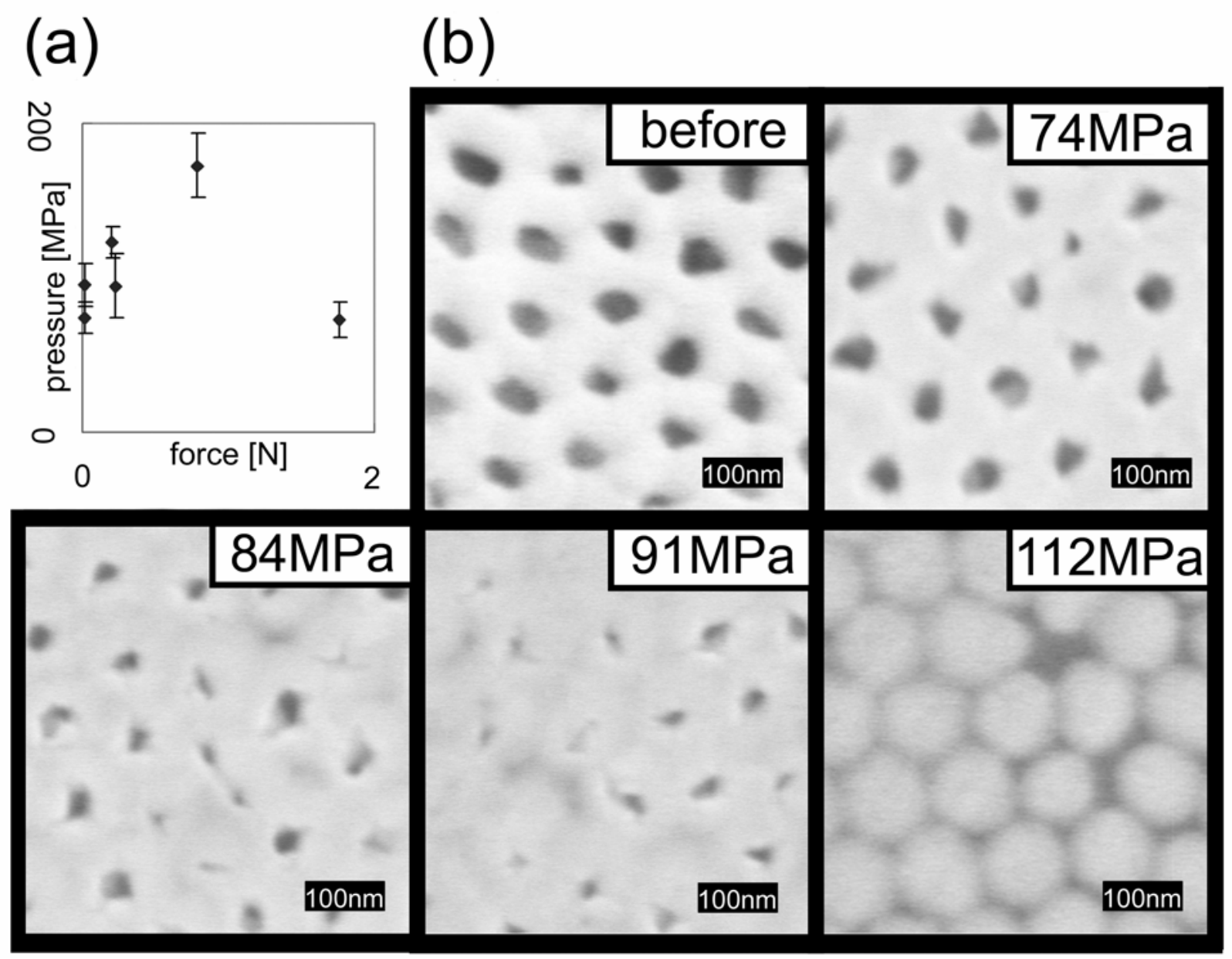

H. Ito and S. Nomura, Fabrication of highly ordered arrays of nanoparticles by mechanical process. 
Fig. 4 (double sized)

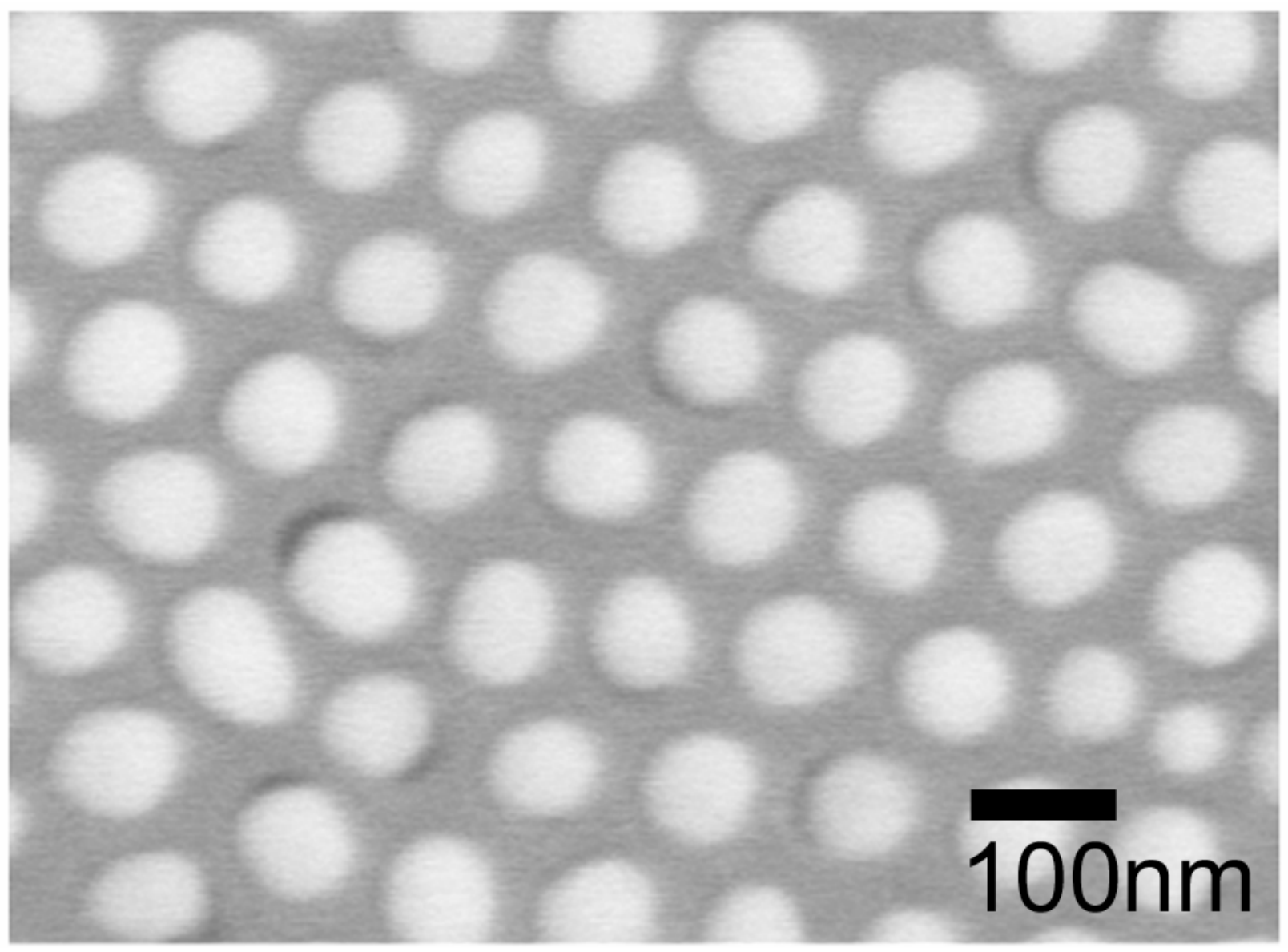

H. Ito and S. Nomura, Fabrication of highly ordered arrays of nanoparticles by mechanical process. 